\title{
Effect of residence time on the efficacy of antidandruff shampoos
}

\author{
C. Piérard-Franchimont*, E. Uhoda*, G. Loussouarn $\dagger$, D. Saint-Léger $\dagger$ and G. E. Piérard \\ ${ }^{*}$ Department of Dermatopathology, Unit of Dermocosmetology, University Hospital Sart Tilman, B-4000 Liège, Belgium, \\ and †L’Oréal Recherche Clichy, F-92583 Clichy, France
}

Received 13 June 2003, Accepted 30 July 2003

Keywords: antifungal, dandruff, lingering effect, Malassezia spp., shampoo

\section{Synopsis}

Dandruff is known to be controlled by fungistatic shampoos active against Malassezia spp. These products also remove the loosely attached scales. This study was performed to assess the effect of a 5-min residence time on the efficacy of antidandruff shampoos. Two commercially available shampoos were used in two groups of 21 panelists with severe dandruff. They contained either $1 \%$ ketoconazole or $1 \%$ piroctone olamine. In each group, intraindividual comparisons were made by a split-scalp design between the effect of a 5-min residence time versus no residence time. Both shampoos induced significant reductions in scaliness and yeast colonization. The beneficial effects were obvious immediately after one single shampooing and 3 days later as well. The improvement was greater with a 5-min residence time. The piroctone olamine treatment benefited more than the ketoconazole treatment from the extension of shampoo-exposure time. In conclusion, the benefit of a residence time in treating dandruff is documented. The level of improvement in efficacy may vary according to the nature of the shampoo.

\section{Résumé}

Il est bien connu que le processus pelliculaire peut être contrôlé par des shampooings aux propriétés fongistatiques actifs contre les levures Malassezia

Correspondence: Prof. G. E. Piérard, Department of Dermatopathology, CHU Sart Tilman, B-4000 Liège, Belgium. Fax: +32 4 3662976; e-mail: gerald.pierard@ulg.ac.be spp. Ces produits doivent aussi éliminer les squames peu adhérentes. Cette étude a été réalisée afin d'évaluer l'effet d'un temps de pose de 5 min lors de l'emploi de shampooings antipelliculaires. Deux shampooings commercialement disponibles ont été utilisés dans 2 groupes de 21 volontaires atteints d'un état pelliculaire important. Ils renfermaient respectivement $1 \%$ de kétoconazole et $1 \%$ de piroctone olamine. Dans chaque groupe, les comparaisons intraindividuelles furent réalisées par la méthode en demi tête entre les effets d'un temps de pose de 5 min par rapport à son absence. Les deux shampooings ont induit une réduction significative de l'état squameux et de la colonization par les levures. Les effets bénéfiques étaient évidents immédiatement après un seul shampooing et persistaient 3 jours plus tard. L'amélioration était plus importante lorsqu'un temps de pose de 5 min avait été respecté. Le traitement par piroctone olamine a bénéficié plus que celui au kétoconazole de l'allongement du temps de contact avec le shampooing. En conclusion, cette étude documente le bénéfice d'un temps de pose pour traiter un état pelliculaire. Le niveau d'amélioration en efficacité peut varier selon la nature du shampooing.

\section{Introduction}

The causative agents of dandruff belong to the group of scalp commensal lipophilic yeasts of the genus Malassezia [1-5]. Microinflammation, parakeratosis and scaling develop around the hair follicle openings [5]. This condition is frequently itchy. Several fungistatic compounds have been shown to improve dandruff condition. The main active agents include imidazole derivatives such as ketoconazole and 
other compounds such as selenium sulphide, zinc pyrithione, piroctone olamine, ciclopirox olamine and tars [5-9]. The ultimate goals of antidandruff products are to remove scales, reduce Malassezia adherence to corneocytes and inhibit the yeast growth.

Most antidandruff products are formulated as shampoos. Conceptually, there is great difference between leave-on and wash-off products when a biological effect is expected on the skin. Indeed, exposure time is one of the parameters affecting the penetration of xenobiotics including active compounds inside the stratum corneum. In addition, extending exposure time to the cleansing mixture helps removing scales, debris and colonizing microorganisms more efficiently. Hence, extending the exposure time is legitimately expected to increase the efficacy of antidandruff shampoos. The exposure time to a shampoo is composed of two successive periods. The first one is the lathering procedure during which active massage is performed. The second phase is facultative and corresponds to the residence time without any mechanical agitation. The latter procedure is generally recommended to dandruff sufferers, but the benefit has not been objectively quantified.

The objective of the study was to assess the impact of exposure time of antidandruff shampoos on efficacy.

\section{Volunteers and methods}

This study was conducted according to the tenets of Good Clinical Practice. Otherwise, healthy volunteers entered in the study if they had short hair styling and clinical signs and symptoms of severe dandruff restricted to the scalp. As an inclusion criterion, partly adhering scales giving an irregular whitish appearance had to cover more than $50 \%$ of both right and left sides of the scalp. Prior to entry in the study, age and sex of the dandruff sufferers were recorded, together with details about duration of dandruff condition, the usual shampoo used and other hair cosmetic habits.

During a 2-week wash-out period, the panelists were asked to use only regular mild shampoo three times per week. At the end of pre-treatment, volunteers came back to the laboratory to enter the assessment procedure. Two randomized groups of 21 subjects were shampooed using either $1 \%$ ketoconazole (shampoo A) or $1 \%$ piroctone olamine (shampoo B). Each subject served as his/her own control by comparing each half-side of the head. The whole scalp was shampooed for $30 \mathrm{~s}$ before entering a splitscalp procedure. A randomized side of the head was thoroughly rinsed at the end of this shampoo application. A 5-min residence time occurred on the other side before rinsing. At the most, a hair blow dryer was used for $1 \mathrm{~min}$.

Dandruff scales were sampled on both temporal sides of the scalp, nearby the back of the ears, using the squamometry method [3-10] at day 1 (D1), just before shampooing and $10 \mathrm{~min}$ after hair drying. Samples were blinded for further analysis. Similar samplings were performed 3 days later (D4). In the interval, no shampoo and hair cosmetics were allowed. Samples were stained by neutral red revealing the living yeasts. They were examined under the microscope, and image analysis was used to quantify their density per square millimetre of scales. Samples were then counterstained for $1 \mathrm{~min}$ by a toluidine blue and basic fuschin solution in order to stain the collected stratum corneum. Reflectance tristimulus colourimetry was performed recording the Chroma $\mathrm{C}^{*}$ value representing the squamometry index as previously described [3-10].

Data had a non-Gaussian distribution according to the Shapiro-Wilk test. Hence, they are presented as medians and ranges. Statistical analysis was performed using paired non-parametric tests. Intraindividual data were compared by the Wilcoxon's matched test. Variations, according to the time of sampling, were compared using the Friedman test followed by the Dunn post-test. Statistical significance was reached when the $P$-value was lower than 0.05. No formal attempt was made to compare statistically the effect of the two shampoos because the number of volunteers was too small for the pre-requisite of an interindividual assessment.

\section{Results}

\section{Squamometry}

The dandruff severity data expressed by the squamometry index are presented in Table I. In both series of panelists, no significant difference in dandruff severity was found between the two randomly allocated sites before shampooing.

Compared to baseline, both tested shampoos decreased dandruff severity immediately and significantly $(P<0.001)$, irrespective of the product-exposure time with the scalp. However, the efficacy was significantly greater at sites where a 5-min residence 
Table I Squamometry index (Chroma C $\left.{ }^{*}\right)$

\begin{tabular}{llrlll}
\hline & \multicolumn{2}{l}{ No development time } & & \multicolumn{2}{c}{ 5-min development time } \\
\cline { 2 - 3 } \cline { 5 - 6 } Evaluation time & Shampoo A & Shampoo B & & Shampoo A & Shampoo B \\
\hline D1 (before shampoo) & $14.3(11.6-17.4)$ & $14.5(10.9-19.6)$ & & $14.2(11.6-19.3)$ & $14.5(12.2-17.5)$ \\
D1 (after shampoo) & $10.3(6.8-14.7)$ & $9.8(5.4-16.9)$ & & $10.0(3.2-14.7)$ & $8.5(3.2-14.8)$ \\
D4 & $11.8(7.7-15.9)$ & $11.3(7.7-20.2)$ & & $10.0(5.4-16.3)$ & $8.4(5.1-16.4)$ \\
\hline
\end{tabular}

Table II Malassezia yeast density $\left(\mathrm{N} \mathrm{mm}^{-2}\right)$

\begin{tabular}{lrrrr}
\hline & No development time & & 5-min development time \\
\cline { 2 - 3 } & \multicolumn{1}{c}{ Shampoo A } & Shampoo B & Shampoo A \\
\hline Evaluation time & $154(132-210)$ & $162(96-204)$ & $159(98-206)$ \\
D1 (before shampoo) & $115(66-154)$ & $104(61-199)$ & $90(35-145)$ \\
D1 (after shampoo) & $129(83-149)$ & $125(92-188)$ & $96(64-179)$ \\
D4 & & & $77(27-162)$ \\
\hline
\end{tabular}

time was applied with shampoo A $(P<0.01)$ and shampoo B $(P<0.001)$.

Compared to baseline, the reduction in the squamometry index values was maintained 3 days after shampooing. This was evidenced with either shampoo when applying a 5-min exposure time $(P<0.001)$ or not applying a 5 -min exposure time $(P<0.01)$. The beneficial effect of extending the total exposure time was still obvious for shampoo A $(P<0.05)$ and shampoo B $(P<0.001)$.

For both shampoos, no significant differences were yielded between the immediate post-shampoo squa- mometry index values and the corresponding values gained 3 days later.

\section{Malassezia load}

The quantification of Malassezia densities is presented in Table II. At inclusion, no statistical difference was found in Malassezia load of the two sides of the scalp in any of the two series of panelists. A significant positive linear correlation $(r=0.86)$ was yielded between Malassezia density and the squamometry index (Fig. 1).

Figure 1 Correlation between Malassezia density and the squamometry index.

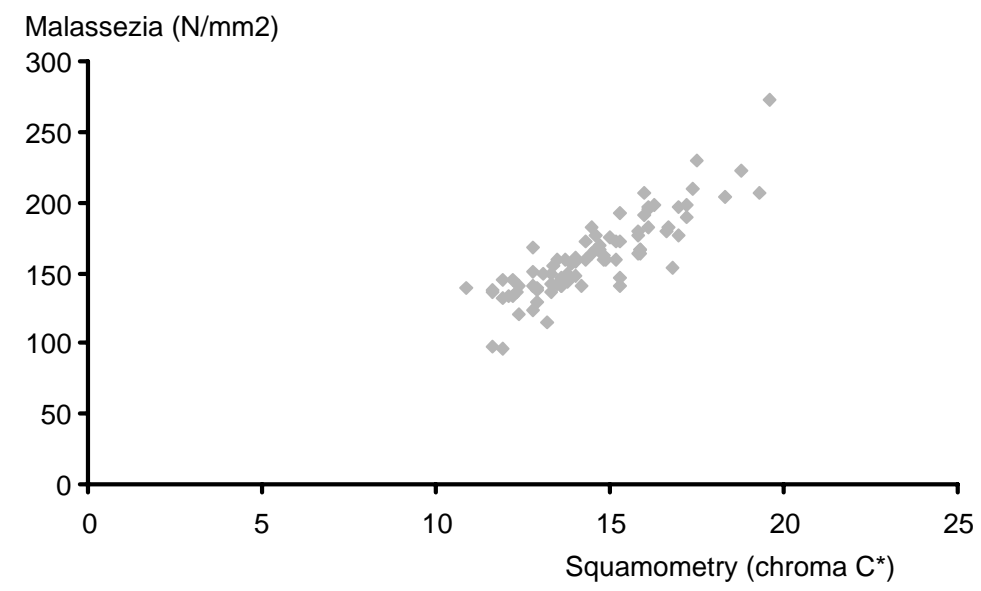


Compared to baseline, the yeast density decreased immediately and significantly with any of the shampoos, irrespective of the application or not of a 5-min residence time. However, the yeast density was much lower $(P<0.001)$ on the sides of the head where the residence time had been utilized for each of the shampoos.

Compared to baseline, the yeast density was still decreased 3 days after shampooing. For shampoo A, the reduction was statistically significant when using $(P<0.001)$ or avoiding $(P<0.01)$ the 5 -min residence time procedure. For shampoo $\mathrm{B}$, the reduction also reached significance when using $(P<0.05)$ or avoiding $(P<0.01)$ the 5 -min residence time. Overall, no difference was found in yeast density between the immediate post-shampoo situation and 3 days later. The only exception was the increase $(P<0.05)$ for shampoo B after 3 days when used with the 5-min development time procedure. The intraindividual difference between both head sides 3 days after shampooing showed that the 5-min residence time was responsible for a more powerful control of the yeast colonization. Significance was reached for both shampoo A $(P<0.001)$ and shampoo B $(P<0.01)$.

\section{Discussion}

Washing hair with a regular shampoo can produce a small and transient effect on dandruff by removing loosely adherent scales. However, the relapsing nature of the scalp condition leads to rapid worsening after shampoo application. The antidandruff efficacy of shampoos exhibiting fungistatic properties is well documented [5]. By contrast, the benefit of including a residence time before rinsing has not been thoroughly studied previously.

The expectation of improving the efficacy of antidandruff shampoos by extending the exposure time must be balanced with the risk of increasing surfactant-induced irritation. This can be predicted by the ex vivo corneosurfametry bioassay [11].

The split-scalp design of the present study with blinded laboratory evaluations allowed the direct comparison of two exposure times (30 s and $5 \mathrm{~min}$, $30 \mathrm{~s})$ using two commercially active antidandruff shampoos. Data collected immediately before and after shampooing were strictly controlled to avoid cross-contamination. Data gathered 3 days later could not exclude possible cross-contamination between both sides of the head. However, the location of the test sites at the back of the ears minimized the risk as much as possible.
The squamometry method was performed on blinded samples in order to quantify objectively the amount of collected dandruff scales. Both shampoos proved their efficacy, irrespective of whether a 5-min residence time was used or not. Squamometry data collected immediately after shampooing measured the efficacy of the shampoos in removing the loosely attached scales. Data collected 3 days later aimed at evaluating the relapse trend in the scaling process. Both tested shampoos showed immediate and lasting efficacy in the control of dandruff. Shampoo B benefited more than shampoo A from the 5-min exposure time. We frame, as an hypothesis, that the benefit of extending the total exposure time corresponding to lathering and residence time is inversely correlated to the binding ability of the actives of the stratum corneum and inside the hair infundibulum. One would also speculate that particle-based actives might be less sensitive to residence time if their deposition at the skin surface is a rapid process.

The living yeast density on the scales was quantified to evaluate the impact of Malassezia colonization in dandruff. It was previously reported that dandruff severity correlated with the Malassezia load [4]. The present findings at inclusion are in line with this concept. Data collected immediately after shampooing showed a consistent decrease in yeast density as previously shown [3,4]. This suggests that Malassezia yeasts were likely detached from superficial corneocytes during shampooing. Another possibility is that dandruff particles removed by the shampoos were more heavily loaded in yeasts than those remaining on the scalp. Data collected 3 days after shampooing were interpreted in terms of efficacy of the antifungals to limit yeast proliferation and recolonization of the scalp. Both shampoos showed efficacy on this microbiological parameter because the yeast density was consistently lowered compared to baseline. A trend for better reducing the Malassezia load was found when a 5-min development time had been respected with either shampoo.

In sum, this study supports the efficacy in treating dandruff with shampoos enriched in appropriate fungistatic compounds, using a 5-min residence time compared favourably with simple shampooing. This differential effect might be amplified by the repeated shampoos. The total exposure time to shampoo and, particularly, the residence time following lathering might influence differently the antidandruff performance according to the shampoo composition. This possibility is worth further investigation. 


\section{Acknowledgements}

This work was supported by the Fonds d'Investissement de la Recherche Scientifique of the University Hospital of Liège.

\section{References}

1. Shuster, S. The aetiology of dandruff and the mode of action of therapeutic agents. Br. J. Dermatol. 111, 235242 (1984).

2. Saint Léger, D., Kligman, A.M. and Stoudemayer,T.Y.The role of the resident microflora in the pathogenesis of dandruff. J. Soc. Cosmet. Chem. 40, 109-117 (1989).

3. Piérard, G.E., Arrese, J.E., Piérard-Franchimont, C. and De Doncker, P. Prolonged effect of antidandruff shampoos. Time to recurrence of Malassezia ovalis colonization of skin. Int. J. Cosmet. Sci. 19, 111-117 (1997).

4. Piérard-Franchimont, C., Arrese, J.E., Durupt, G., Ries, G., Cauwenbergh, G. and Piérard, G.E. Correlation between Malassezia spp. load and dandruff severity. J. Mycol. Med. 8, 83-86 (1998).

5. Piérard-Franchimont, C., Hermanns, J.F., Degreef, H. and Piérard, G.E. From axioms to new insights into dandruff. Dermatology 200, 93-98 (2000).
6. Loden, M. and Wessman, C. The antidandruff efficacy of a shampoo containing piroctone olamine and salicyclic acid in comparison to that of a zinc pyrithione shampoo. Int. J. Cosmet. Sci. 22, 285-289 (2000).

7. Piérard-Franchimont, C., Arrese, J.E. and De Doncker, P. Effect of ketoconazole $1 \%$ and $2 \%$ shampoos on severe dandruff and seborrhoeic dermatitis: clinical, squamometric and mycological assessments. Dermatology 202, 171-176 (2001).

8. Piérard-Franchimont, C., Goffin, V., Henry, H., Uhoda, I., Braham, C. and Piérard, G.E. Nudging hair shedding by antidandruff shampoos. A comparison of $1 \%$ ketoconazole, $1 \%$ piroctone olamine and $1 \%$ zinc pyrithione formulations. Int. J. Cosmet. Sci. 24, 249-256 (2002).

9. Piérard-Franchimont, C., Goffin, V., Decroix, J. and Piérard, G.E. A multicenter randomized trial of ketoconazole $2 \%$ and zinc pyrithione $1 \%$ shampoos in severe dandruff and seborrheic dermatitis. Skin Pharmacol. Appl. Skin Physiol. 15, 434-441 (2002).

10. Piérard-Franchimont, C., Henry, F. and Piérard, G.E.The SACD method and the XLRS squamometry tests revisited. Int. J. Cosmet. Sci. 22, 437-446 (2000).

11. Goffin, V.V.C., Piérard-Franchimont, C. and Piérard, G.E. Antidandruff shampoos and the stratum corneum. J. Dermatol.Treat. 7, 223-225 (1996). 